\title{
Implementing Virtual Buffer for Electric Power Grids
}

\author{
Rong Gao and Lefteri H. Tsoukalas \\ Applied Intelligent Systems Lab, School of Nuclear Enigineering, Purdue University, \\ 400 Central Drive, West Lafaytte, IN, USA \\ \{gao, tsoukala\}@ecn.purdue.edu
}

\begin{abstract}
The electric power grid is a vital network for every aspect of our life. The lack of buffer between generation and consumption makes the power grid unstable and fragile. While large scale power storage is not technically and economically feasible at present stage, we argue that a virtual buffer could be effectively implemented through a demand side management strategy built upon the concept of dynamic data driven paradigm. An intelligent scheduling module implemented inside a smart meter enables customers to access electricity safely and economically. The managed use of electricity acts effectively as a buffer to provide the much needed stability for the power grid. Pioneering efforts intending to implement these concepts have been conducted by groups such as the Consortium for the Intelligent Management of Electric Grid. Progresses have been made and reported in this paper.
\end{abstract}

Keywords: Electric Power Grid, Artificial Intelligence, Dynamic Data Driven.

\section{Introduction}

The electric power grid is a vital network for every aspect of our life. The grid is so complex that it experiences the same instability problem as other complex systems do [1]. The system can ran into an unstable point without notice until a large scale collapse spreads, such as the major northeast blackout of 2003. The fragile nature of the electric power grid, to a great extend, has to do with the fact that electricity can not be stored in the grid. Electricity is consumed when it is generated. The lack of cushion between generation and consumption constitutes a major unstable factor for the power system.

On the other hands, it is helpful to study another example of complex system, the Internet. The Internet connects billions of users in the world. The degrees of connectivity and irregularity are enormous. Yet surprisingly high stability is achieved considering the openness provide by the Internet. Users have the maximum freedom to use the resources with little restriction, something that is hard to imagine for the electric power grid. The key behind this impressive performance is the protocols, a set of rules that very player agrees to. Protocols regulate the creation, transfer and use of the resources. The Internet protocols roots on the assumption that very one has equal access to the resources on the network. This principle of fairness is the guideline for resolving conflict originated from the competition of resources. The protocols are the reason why the Internet is able to maintain functional under malignant environment. 
The protocols are feasible and enforceable because of one big assumption that the resource (information) can be stored somewhere in the network. This assumption is the cornerstone for many key concepts in the protocols, such as routing and conflict resolving. In the Internet, resources are generated, stored, and consumed. The lag between generation and consumption ranges from milliseconds to seconds or minutes depending on the traffic in the network. The delay has proven an important stabling factor for the network.

Having had a successful case at hand, it is natural to ask whether we can duplicate the same kind of success in the electric power grid. As the initial attempts, we can try to develop protocols similar to the ones used in the Internet to regulate the generation, transportation, and consumption of the electricity. This is certainly unrealistic for the current power grid. However, as the discussion and research on smart power meter have been going for a while, we have the reason to believe that in the near future every household will have one of these intelligent device installed. The intelligent meter is connected to the network and is able to communicate with the outside world such as the electricity supplier. The meter will have the computing capability, making possible the implementation of a set of fairy complicated protocols. With these smart meters at our disposal, there is only one thing between us and the Internet-type stable electric power grid - we need the means to store electricity. There have been lots of efforts being spent on electricity storage. Some successes have been reported [2]. But the solution for large scale storage that can make a big impact to the power grid is still not technically and economically feasible. In this paper, we argue that through the utilization of information technologies, we can implement a virtual buffer between the generation and consumption without the need to physically store the electricity in the grid.

\section{Virtual Buffer for Electric Power Grid}

As we know, the lack of buffer between generation and consumption is the source of problem for many complex systems such as the electric power grid. Even though we don't have a feasible way to physically store large amount of electricity in the network so far, we are able to mimic the effect of storage through a virtual buffer.

A virtual buffer between generation and consumption can be created through demand side management strategy which is build upon the practices of dynamic data driven paradigms. With the emergence of intelligent meters, it is possible to dynamically schedule the use of electricity of every customer. This dynamic scheduling will create a sheet of virtual buffer for generation and consumption as we argue here. Under the new paradigm, the consumption of electricity of every customer is intelligently managed. Customers don't power up their electricity-hungry machines at will. Rather, they make smart decisions after balancing the costs and benefits. For example, some non-urgent activities, such as laundry, can be scheduled to sometime during the day when electricity is abundant and cheap. The costs of the electricity are determined by the supply-to-demand ratio and the capacity of the network to transfer the resources. This managed use of resources is analogous to the access control widely used in the Internet. A buffer between generation and consumption is therefore created, virtually. No physic laws are broke. The electricity is still actually consumed 
when generated. However, from the customer point of view, with dynamic consumption scheduling the resources (electricity) are created and then stored somewhere in the power grid before they are used. The analogy is shown in Fig. 1. The virtual buffer can greatly increase the stability of the power grid.
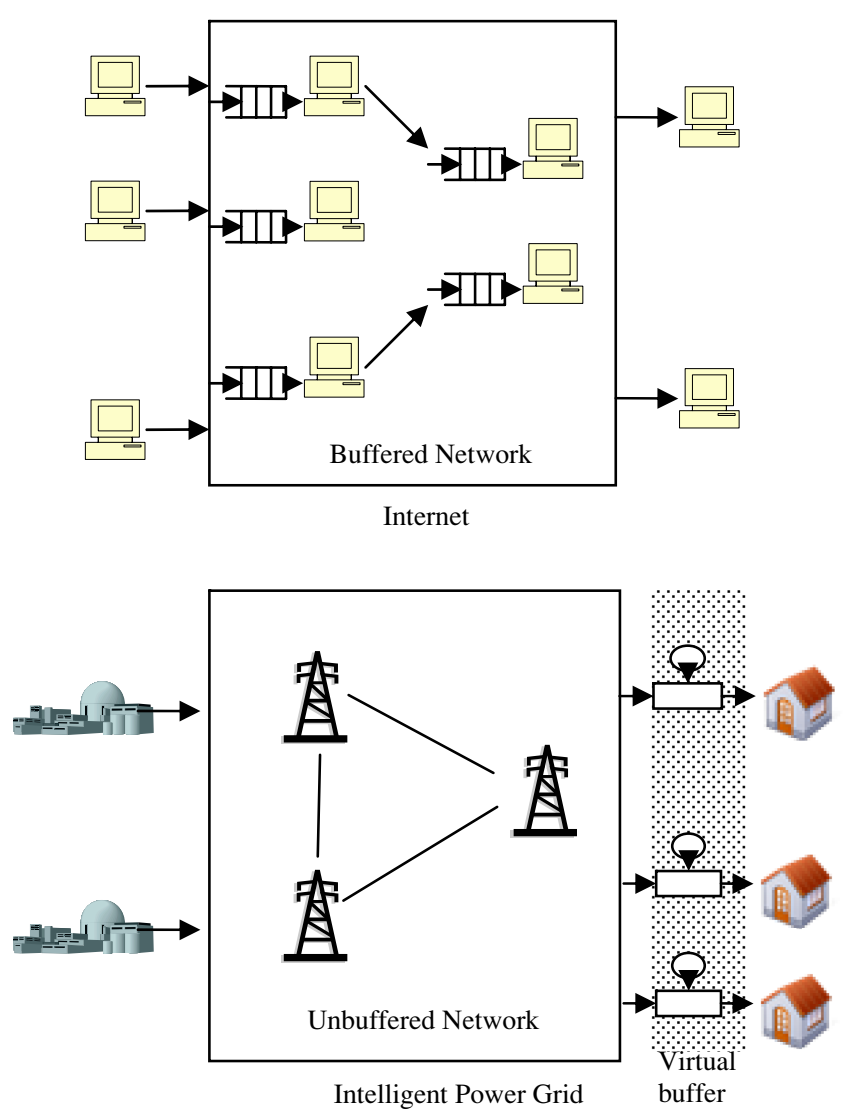

Fig. 1. Internet and Power Grid with Virtual Buffer

Dynamic scheduling has to be carried out by software agents or intelligent agents. The intelligent agents will act on behalf of their clients, making reasonable decision based the analysis of the situation. One of the most important analysis powers an agent has to possess is the anticipation capability. The intelligent agent needs to predict its client's future consumption pattern to make scheduling possible. In other words, load forecasting capability is the central piece for such a system. A pioneering effort, conducted by CIMEG, has recognized this need and advanced many techniques to make that possible. 


\section{CIMEG and TELOS}

In 1999, EPRI and DOD funded the Consortium for the Intelligent Management of the Electric Power Grid (CIMEG) to develop intelligent approaches to defend power systems against potential threats [3]. CIMEG was led by Purdue University and included partners from The University of Tennessee, Fisk University, TVA and ComEd (now Exelon). CIMEG advanced an anticipatory control paradigm with which power systems can act proactively based on early perceptions of potential threats. It uses a bottom-up approach to circumvent the technical difficulty of defining the global health of power system at the top level, ash shown in Fig. 2. The concept of Local Area Grid (LAG) is extremely important in CIMEG. A LAG is a demand-based autonomous entity consisting of an appropriated mixture of different customers, charged with the necessary authority to maintain its own health by regulating or curtailing the power consumption of its members. Once all LAGs have achieved security, the whole grid, which is constructed by a number of LAGs in a hierarchical manner, achieves security as well. To pursue the health of a LAG, intelligent agents are used. Intelligent agents monitor every load within the LAG, forecasting the power consumption of each individual load and taking anticipatory actions to prevent potential cascade of faults. A prototypical system called the Transmission Entities with Learning-capabilities and Online Self-healing (TELOS) has been developed and implemented in the service area of Exelon-Chicago and Argonne National Laboratory [4].

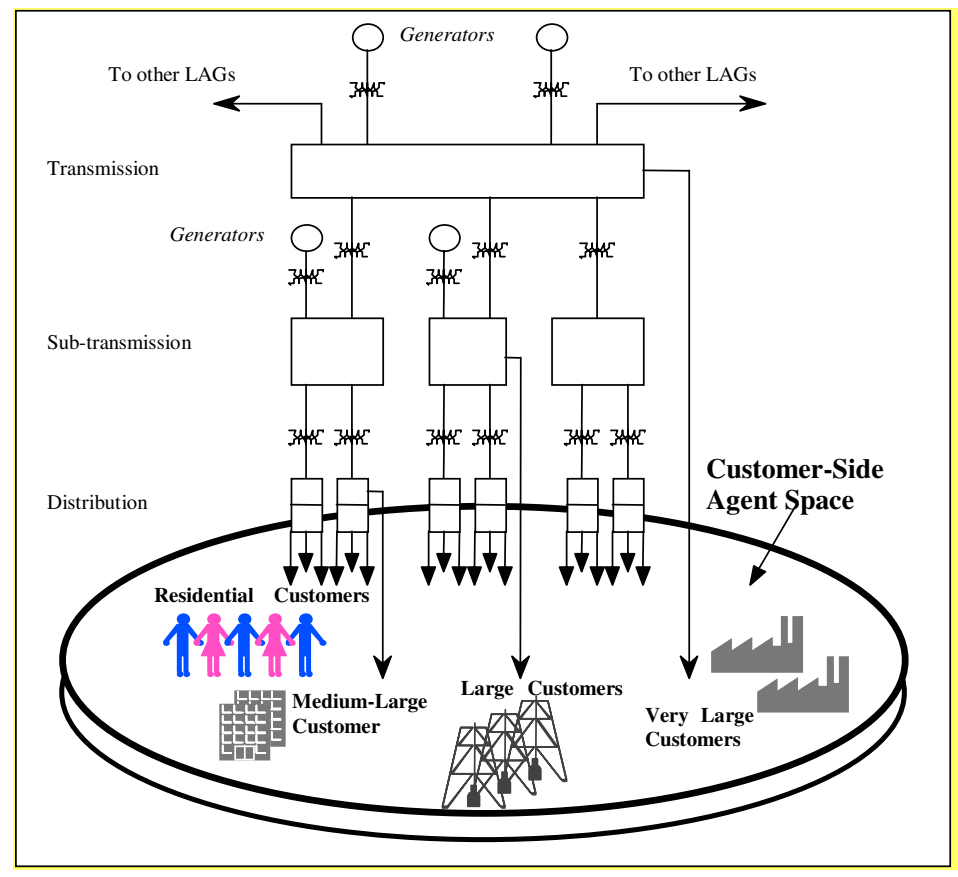

Fig. 2. CIMEG's picture of electric power grid 
CIMEG's mission is to create a platform for managing the power grid intelligently. In CIMEG's vision, customers play more active role than in current power system infrastructure. Lots of solicitation and negotiations are involved, as shown in Fig. 3. The customer, who is represented by an intelligent meter in Fig. 3, predicts its need for the electricity and place order in the market. The amount of the order is influenced by the market price of the electricity, which is further determined by the difference of the demand and supply and also the capacity of the network. Economic models with price elasticity are used in the process [5]. The active interaction between customers and suppliers create a virtual buffer between consumption and generation as discussed earlier.

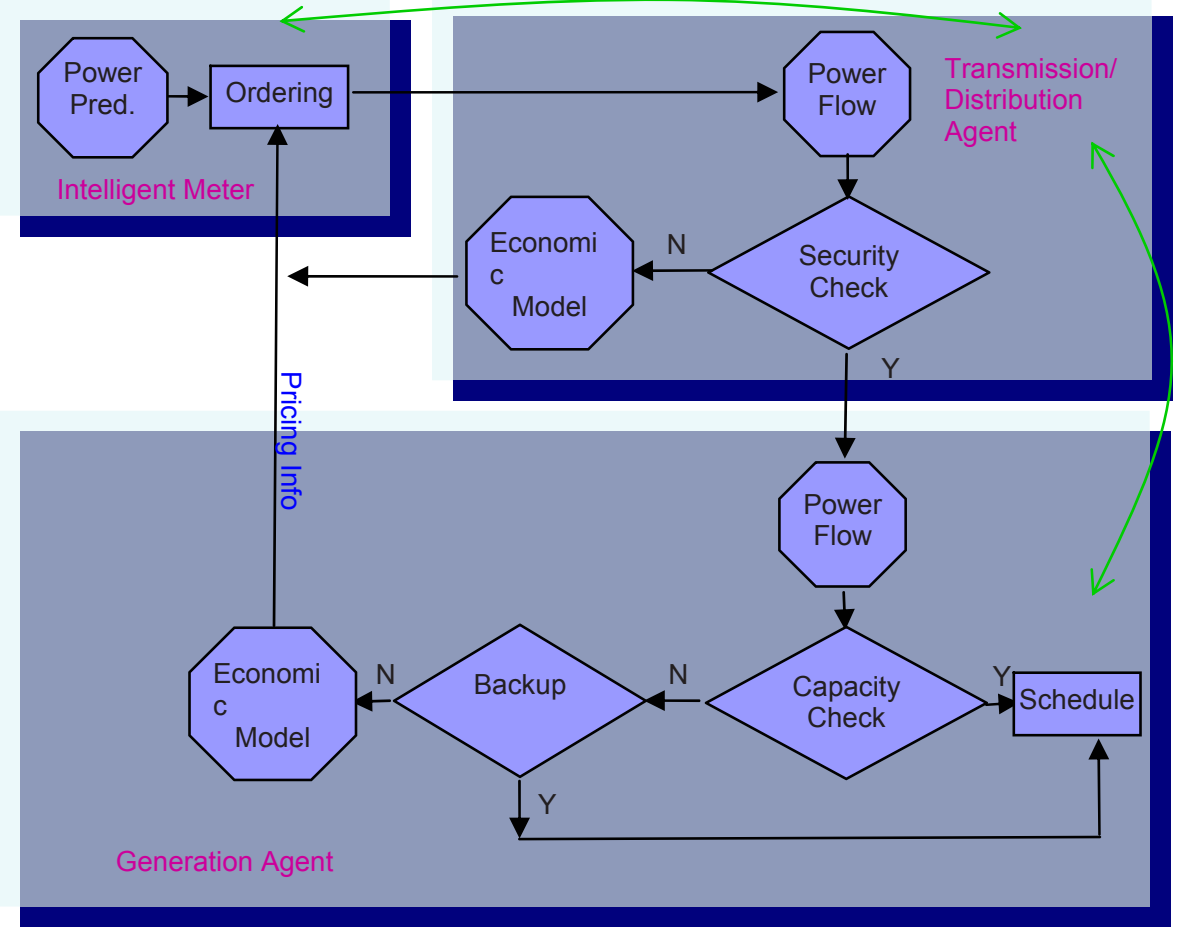

Fig. 3. Interactions between customers and suppliers

One of the key assumptions taken by CIMEG is that the demand of customers can be predicted to a useful degree of accuracy. Among many existing prediction algorithms, neural networks outperform others because of their superb capabilities in handling non-linear behaviors [6].

TELOS achieves improved forecasting accuracy by performing load forecast calculations at the customer site. The customer is capable of tracking local weather conditions as well as the local load demand. TELOS forecasts are based on neurofuzzy algorithms, which are highly adaptive to changing conditions. Preliminary results show that simple feed forward neural networks are well suited to predicting load at the customer scale. Fig. 4 shows neural-network-based load prediction for a large commercial customer. 


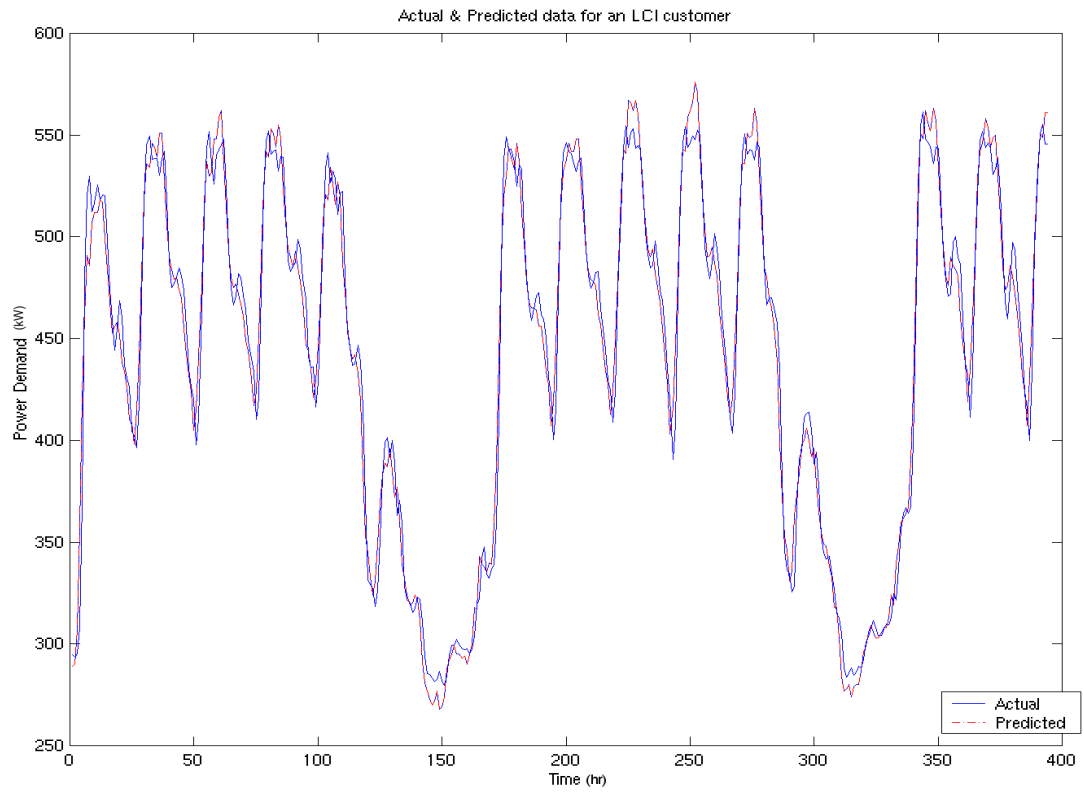

Fig. 4. Neural-network-based load forecasting for actual commercial customer
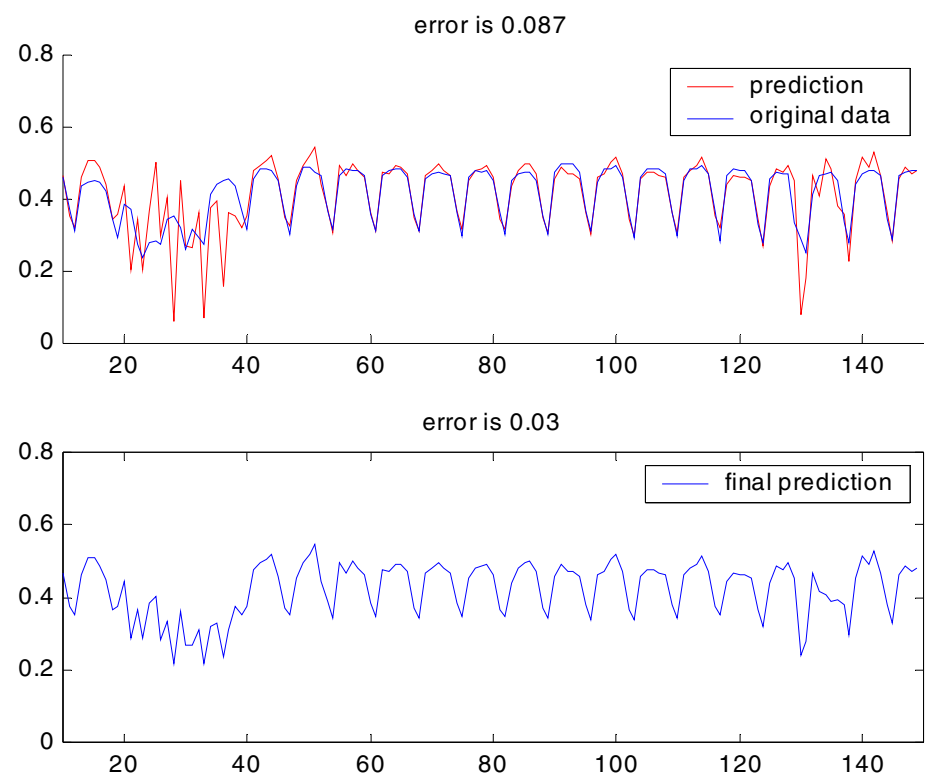

Fig. 5. The prediction results with (bottom) and without (upper) compensations 
Neural networks are well qualified for handling steady state cases, wherever typical behaviors have been seen and generalized. Some unexpected events such as favorite sport programs seriously cause enormous error in prediction. CIMEG has developed a fuzzy logic module to process such situation. This module, called PROTREND, quickly detects any deviation from the steady state and generates a compensation for the final prediction. The results in Fig. 5 show a consumption pattern over a winter break. PROTREND effectively compensates a large portion of errors and makes the final prediction results more reliable.

\section{Conclusions and Future Work}

A regulation between generation and consumption is vital for the stability of a complex system with limited resources. An effective way for regulation is to create buffers where resources can stored when not needed and released when requested. We have shown that an effective form of buffer can be simulated with the intelligent management of the consumption when no means of storage is available. CIMEG has made some promising results, demonstrating the feasibility of intelligent management based on the anticipation of consumption. Accurate load forecasting is possible. However, CIMEG's demo was implemented on a very small system. Large scale demonstrations are necessary, both on simulation and theoretical analysis.

\section{Acknowledgments}

This project is partly supported by the National Science Foundation under contract no 0540342-CNS.

\section{References}

1. Amin, M.:Toward Self-Healing Energy Infrastructure Systems. In: IEEE Compouter Applications in Power, Volume 14, Number 1, 220-28 (2001)

2. Ribeiro, P. F., et al: Energy Storage Systems for Advanced Power Application.In: Proceedings of the IEEE, Vol 89, Issue 12, p1744-1756,(2001)

3. CIMEG, Intelligent Management of the Power Grid: An Anticipatory, Multi-Agent, High Performance Computing Approach, EPRI, Palo Alto, CA, and Applied Intelligent Systems Lab, School of Nuclear Engineering, Purdue University, West Lafayette, IN, 2004

4. Gao, R., Tsoukalas, L. H.:Anticipatory Paradigm for Modern Power System Protection. In:ISAP, Lemnos, Greece (2003)

5. Gao, R., Tsoukalas, L. H.: Short-Term Elasticities via Intelligent Tools for Modern Power Systems,.In: MedPower'02, Athens, Greece(2002)

6. Tsoukalas, L., Uhrig, R. : Fuzzy and Neural Approaches in Engineering. New York: Wiley, (1997) 